\title{
ON FINITELY GENERATED FLAT MODULES III
}

\author{
S. JØNDRUP
}

\section{Introduction.}

Let us first recall some definitions from [3]. A ring $R$ is said to be a left- $n$-FGFP ring (right- $n$-FGFP ring) if any $n$-generated flat left (right) $R$-module is projective. $R$ is called an $n$-FGFP ring if $R$ is a left- and right$n$-FGFP ring. If $R$ is a right- $n$-FGFP for all $n$, then we say that $R$ is a right-FGFP ring. A right- and left-FGFP ring is called an FGFP ring. We always assume that $n$ is finite. For $n$ equal to $x_{0}$ a left- $n$-FGFP ring is left perfect [1].

It is well-known that a semiperfect ring is an FGFP ring, ef. J. Lambek $[4, \S 5.4$, exercise 10]. In [3] it is proved that a subring of a right noetherian ring is an FGFP ring and it is proved that subrings of left- $n$ FGFP rings are left- $n$-FGFP rings, too. This last theorem was also proved by I. I. Sahaev [6].

In section 1 we shall prove that a ring $R$, with w.gl.dim. $R \leqq 1$, is a left- $n$-FGFP ring if and only if $R$ is a right- $n$-FGFP ring. As a corollary of the proof we get a new and simple proof of the fact that any $n$-fir is an $n$-FGFP ring.

In section 2 we construct a ring $R$ with the following properties:

(i) There exists a cyclic flat non-projective left $R$-module.

(ii) $R$ is a right-1-FGFP ring.

All rings considered in this note are associative, with 1 , all ring homomorphisms preserve 1 and subrings have the same 1 .

\section{Left- and right- $n$-FGFP rings.}

In the study of left-n-FGFP rings the following lemma is useful:

Lemma 1.1. Let $R$ be any ring. Then the following conditions are equivalent:

(i) Any cyclic flat left $R$-module is projective.

(ii) Any ascending chain of principal left ideals

terminates.

$$
\left(a_{1}\right) \leqq \ldots \subseteq\left(a_{m}\right) \subseteq \ldots, \text { where } a_{m} a_{m+1}=a_{m},
$$


(iii) Any descending chain of principal right ideals

terminates.

$$
\left(a_{1}\right) \supseteq \ldots \supseteqq\left(a_{m}\right) \supseteqq \ldots, \text { where } a_{m} a_{m+1}=a_{m+1},
$$

The lemma is proved by I. I. Sahaev in [5].

Furthermore we need the following well-known lemma:

LEMMA 1.2. The following statements are equivalent for the ring $R$ :

(i) $R$ has no infinite set of non-zero orthogonal idempotents.

(ii) $R$ satisfies the ascending chain condition on ideals $e R$ (or ideals $R e$ ), where e denotes an idempotent in $R$.

(iii) $R$ satisfies the descending chain condition on ideals e $R$ (or ideals $R e$ ), where $e$ denotes an idempotent in $R$.

Lemma 1.3. Suppose $R$ satisfies the ascending chain condition on left point annulets, that is, ideals of the form $l\{a\}=\{x \mid x a=0\}$, then any cyclic flat left $R$-module is projective.

Proof. It is easily checked that $R$ satisfies the equivalent conditions in lemma 1.2.

Suppose we are given an ascending chain of principal left ideals

$$
\left(a_{1}\right) \subseteq \ldots \subseteq\left(a_{m}\right) \leqq \ldots, \quad \text { with } \quad a_{m} a_{m+1}=a_{m} .
$$

We have to prove that (1) becomes stationary. We claim that $l\left\{\left(1-a_{m}\right)\right\} \subseteq l\left\{\left(1-a_{m+1}\right)\right\}$ for all $m$. If $x$ is an element in $l\left\{\left(1-a_{m}\right)\right\}$, then $x-x a_{m}=0$. Hence $x a_{m+1}-x a_{m} a_{m+1}=0$ and consequently

$$
x a_{m+1}=x a_{m}=x, \text { that is, } x \in l\left\{\left(1-a_{m+1}\right)\right\} .
$$

It follows now that there exists an $m_{0}$ such that

$$
l\left\{\left(1-a_{m}\right)\right\}=l\left\{\left(1-a_{m_{0}}\right)\right\}
$$

for all $m \geqq m_{0}$. In particular if $m \geqq m_{0}+1$, then

$$
l\left\{\left(1-a_{m}\right)\right\}=l\left\{\left(1-a_{m-1}\right)\right\} .
$$

Now $a_{m-1} \in l\left\{\left(1-a_{m}\right)\right\}$, hence $a_{m-1} \in l\left\{\left(1-a_{m-1}\right\}\right\}$, that is, $a_{m-1}$ is an idempotent. Thus, $a_{m}$ is idempotent for all $m \geqq m_{0}$, and the proof of lemma 1.3 is completed.

A similar argument will show that the cyclic flat left modules of a ring with descending chain condition on right point annulets are projective.

For a later purpose we need the left-right symmetric of lemma 1.3. 
LEMMA 1.4. If the ring $R$ satisfies the ascending chain condition on right point annulets or if $R$ satisfies the descending chain condition on left point annulets, then any cyclic flat right module is projective.

Proposition 1.5. Let $R$ be a ring with no infinite set of orthogonal idempotents. If the principal left ideals in $R$ are projective, then $R$ is a 1-FGFP ring.

Proof. Since all left point annulets are generated by idempotents, it follows from lemma 1.2 that $R$ satisfies the ascending and descending chain conditions on left point annulets. Thus, by lemma 1.3 and lemma $1.4, R$ is a 1 -FGFP ring.

Morita technique gives the next results.

COROLLARY 1. If all n-generated left ideals of the ring $R$ are projective and if $R_{n}$ (the ring of $(n \times n)$-matrices over $R$ ) has no infinite set of non-zero orthogonal idempotents, then $R$ is an n-FGFP ring.

Corollary 2. Assume w.gl.dim. $R \leqq 1$. Then $R$ is a left-n-FGFP ring if and only if $R$ is a right- $n$-FGFP ring.

Proof. It suffices to prove corollary 2 for $n=1$. If $R$ is a left-1-FGFP ring, then the principal left ideals of $R$ are projective. It follows from lemma 1.1 and lemma 1.2 that $R$ has no infinite set of non-zero orthogonal idempotents. Corollary 2 follows now immediately.

Corollary 3 (cf. [2] and [3]). Any n-fir is an n-FGFP ring.

Proof. It is well known or readily checked that the ring of $(n \times n)$ matrices over an $n$-fir satisfies the ascending chain condition on left and right point annulets.

REMARK. As a consequence of corollary 2 let us note that a left semihereditary ring for which the ring of $(n \times n)$-matrices for all $n$ has no infinite set of non-zero orthogonal idempotens is right semihereditary (cf. L. W. Small [7]).

Proposition 1.6. If any left or right zero divisor in $R$ is nilpotent, then $R$ is a 1-FGFP ring. 
Proof. Assume we have a chain of principal left ideals

$$
\begin{aligned}
& \ldots \leqq\left(a_{-n}\right) \leqq \ldots \leqq\left(a_{1}\right) \leqq \ldots \leqq\left(a_{n}\right) \leqq \ldots, \\
& \text { where } \quad a_{m}=a_{m} a_{m+1}
\end{aligned}
$$

for all $m \in \mathbf{Z}$. It suffices to prove that (2) becomes stationary. We can assume that

$$
(0) \neq\left(a_{m}\right) \neq R \quad \text { for all } m \text {. }
$$

The equation $a_{m}\left(1-a_{m+1}\right)=0$ shows that $a_{m}$ or $1-a_{m}$ is nilpotent for all $m$. If $a_{m}$ is nilpotent, then

$$
a_{m-1}=a_{m-1} a_{m}=\ldots=a_{m-1} a_{m}^{s}=0
$$

for $s$ suitably large, and we are done in this case. If $\left(1-a_{m}\right)$ is nilpotent for all $m$, then the equation $\left(1-a_{m}\right)\left(1-a_{m+1}\right)=\left(1-a_{m+1}\right)$ shows that $a_{m+1}=1$. The proof of the proposition is now completed.

\section{An example.}

An example of a ring, $R$, with any cyclic flat right module projective and with a cyclic flat non-projective left module is given as follows.

Let $K$ be any commutative field. We take $R$ to be the $K$-algebra on the generators $X_{i}, i=1,2, \ldots$, and defining relations

$$
X_{i} X_{i+1}=X_{i}, \quad i=1,2, \ldots .
$$

From lemma 1.1 it follows that there exists a cyclic flat non-projective left $R$-module. The left-right symmetric to lemma 1.1 shows that $R$ is a right-1-FGFP ring if and only if any ascending chain of principal right ideals

$$
\left(a_{1}\right) \subseteq \ldots \subseteq\left(a_{m}\right) \subseteq \ldots, \quad \text { with } \quad a_{m} a_{m-1}=a_{m-1},
$$

terminates. It is not hard to check that any element in $R$ can be written as $k+a, k \in K$ and $a$ of the form $\sum k_{\gamma_{r} \ldots \gamma_{1}} X_{r}^{\gamma_{r}} \ldots X_{1}^{\gamma_{1}}$, where in no term all the $\gamma_{j}$ are zero, the $r$-tuples $\left(\gamma_{r}, \ldots, \gamma_{1}\right)$ are all different, and all $k_{\gamma_{r} \ldots \gamma_{1}}$ are different from zero. This representation is unique. Each term $X_{r}^{\gamma_{r}} \ldots X_{1}^{\gamma_{1}}$ is called a monomial in the representation of $a$.

Let us first assume that all the $k_{i}$ which appear as constant terms in the expressions of the $a_{i}$ in (3) are zero. We can furthermore assume that $a_{1}$ is non-zero. Choose $p$ maximal with respect to the existence of a monomial of the form $X_{r}^{\gamma_{r}} \ldots X_{p}^{\gamma_{p}}$ in $a_{1}$. Consider all these monomials in the representation of $a_{1}$ and fix one with $\left(\gamma_{p}, \ldots, \gamma_{r}, 0, \ldots\right)$ minimal in lexicographical order. From the minimality of $\left(\gamma_{p}, \ldots, \gamma_{r}, 0, \ldots\right)$ it follows that the corresponding minimal monomial never appears as a monomial in an expression of the form $a_{2} a_{1}$. 
We can now assume that infinitely many of the $k_{i}$ in (3) are non-zero. It suffices to consider the case, where all the $k_{i}$ are non-zero.

If $a_{i}=k_{i}+b_{i}$ with $k_{i} \in K$ and $b_{i}$ a $K$-linear combination of monomials, then the relations (3) become

$$
k_{i} k_{i-1}=k_{i-1} \quad \text { and } \quad b_{i} k_{i-1}+b_{i} b_{i-1}+k_{i} b_{i-1}=b_{i-1} .
$$

Thus, we conclude that $k_{i}=1$ for all $i>1$. Hence for $i \geqq 2$

$$
b_{i+1} b_{i}=-b_{i+1} .
$$

Let us assume that these equations hold for all $i$. In $b_{1}$, which is nonzero, let us choose a monomial $X_{r}^{\gamma_{r}} \ldots X_{1}^{{ }^{\gamma_{1}}}$ such that $\left(\gamma_{1}, \ldots, \gamma_{r}, 0, \ldots\right)$ is minimal in lexicographical order. Suppose

$$
\gamma_{1}=\ldots=\gamma_{p}=0 \text {. }
$$

The equation $b_{3} b_{2}=-b_{3}$ shows that there exists a monomial of the form $X_{t}{ }^{\gamma_{t}} \ldots X_{1}^{\gamma_{1}}$ with $\gamma_{1}=0$ in the canonical representation of $b_{2}$. So it follows that all the $b_{i}$ have a monomial with " $\gamma_{1}=0$ ". Again the equation $b_{3} b_{2}=-b_{3}$ shows that $b_{2}$ "has a monomial with $\gamma_{1}=\gamma_{2}=0$ " (look at the terms with " $\gamma_{1}=0$ "). Thus, there exists a monomial in $b_{2}$ of the form $X_{t}^{\gamma_{t}} \ldots X_{n}^{\gamma_{n}}$ for all $n$. In particular $b_{2}$ has a monomial of the form $X_{s}^{\gamma_{s}} \ldots X_{p+1}^{\gamma_{p+1}}$, and this contradicts the equation $b_{2} b_{1}=-b_{2}$.

\section{REFERENCES}

1. H. Bass, Finitistic dimension and a homological generalization of semiprimary rings, Trans. Amer. Math. Soc. 95 (1960), 466-488.

2. C. U. Jensen, Some cardinality questions for flat modules and coherence, J. Algebra 12 (1969), 231-241.

3. S. Jøndrup, On finitely generated flat modules II, Math. Scand. 27 (1970), 105-112.

4. J. Lambek, Lectures on rings and modules, Blaisdell, Waltham, 1965.

5. I. I. Sahaev, The projectivity of finitely generated flat modules, Sibirsk. Mat. Ž. 6 (1965), 564-573. (In Russian.)

6. I. I. Sahaev, About rings for which any finitely generated flat module is projective, Izv. Vysš, Učebn. Zaved. Matematica 9 (88), (1969), 65-73. (In Russian.)

7. L. W. Small, Semihereditary rings, Bull. Amer. Math. Soc. 73 (1967), 656-658. 